\title{
Les protocoles substitutifs en hémostase pour la chirurgie orale
}

\section{Dr Michèle Martin}

Nancy

L'hémostase est un point clé en chirurgie orale avec un enjeu per et post-opératoire. Le risque hémorragique, comme dans d'autres types de chirurgies, est variable selon le geste réalisé. Un certain nombre de gestes sont accessibles à la mise en place de moyens locaux d'hémostase (sutures, éponges hémostatiques, colles, acide tranexamique, ...). Ces moyens efficaces permettent d'ailleurs la poursuite des traitements anti-thrombotiques chez les patients stables et en cas de geste à faible risque hémorragique. En cas de maladie hémorragique constitutionnelle, la réflexion est quelque peu différente. Même les gestes chirurgicaux classés comme étant à faible risque hémorragique doivent en effet être encadrés par un protocole thérapeutique substitutif, généralement d'administration intraveineuse, visant à améliorer sinon normaliser l'hémostase générale, en complément des moyens locaux d'hémostase qui restent indispensables. Les troubles de la coagulation tels que l'hémophilie entraînent en effet des saignements fréquemment retardés et prolongés. On imagine aisément que le taux de facteur de coagulation concerné et le risque hémorragique du geste envisagé suffisent à rédiger ce type de protocole. En réalité le risque hémorragique est variable et parfois peu prédictible pour certains désordres de l'hémostase. Certaines anomalies rares comme les dysfibrinogénémies peuvent même avoir des conséquences thrombotiques plutôt qu'hémorragiques selon la mutation génétique présente. D'autre part, certains traitements substitutifs sont à envisager avec précaution du fait d'un sur-risque thrombotique qui a été décrit comme pouvant être propre au produit ; c'est le cas des concentrés de facteur XI. Nous allons nous appuyer sur quelques expériences cliniques pour expliquer notre réflexion quant à l'encadrement des gestes de chirurgie orale chez ces patients atteints d'anomalies de l'hémostase plus ou moins courantes (hémophilie, maladie de Willebrand, déficit en facteur XI, en facteur VII, hypo et dysfibrinogénémies, thrombopathies). Ainsi, la collaboration entre chirurgiens et hémostasiens est indispensable afin d'optimiser la prise en charge de ces patients. 